\title{
A NOTE ON QUASISYMMETRIC HOMEOMORPHISMS
}

\author{
Shuan Tang and Pengcheng Wu \\ Guizhou Normal University, School of Mathematics Sciences \\ Guiyang 550001, P. R. China; tsaflyhigher@163.com \\ Guizhou Normal University, School of Mathematics Sciences \\ Guiyang 550001, P. R. China; wupc@gznu.edu.cn
}

\begin{abstract}
By means of some integral operators and kernel functions, we characterize when a sense preserving homeomorphism $h$ on the unit circle $S^{1}$ is quasisymmetric, symmetric or $p$ integrable asymptotic affine. As an application, we use these results to characterize the pull-back operator, induced by a quasisymmetric homeomorphism on $S^{1}$.
\end{abstract}

\section{Introduction and results}

Let $\Delta=\{z:|z|<1\}$ be the unit disk in the complex plane $\mathbf{C}, \Delta^{*}=\overline{\mathbf{C}} \backslash \bar{\Delta}$ and $S^{1}=\{z:|z|=1\}$. We say that a sense preserving homeomorphism $h$ on the unit circle $S^{1}$ is quasisymmetric if there is some $M>0$ such that

$$
\frac{1}{M} \leq\left|\frac{h\left(I_{1}\right)}{h\left(I_{2}\right)}\right| \leq M
$$

for all pairs of adjacent arcs on the unit circle $S^{1}$ with the same arc length $\left|I_{1}\right|=\left|I_{2}\right| \leq$ $\pi$. Beurling and Ahlfors gave a very important characterization of quasisymmetric homeomorphism (see [BA]).

Theorem 1.1. [BA] A sense preserving self-homeomorphism $h$ on the unit circle $S^{1}$ is quasisymmetric if and only if there exists some quasiconformal homeomorphism of $\Delta$ onto itself which has boundary value $h$.

In [BA], Beurling and Ahlfors constructed a quasiconformal extension of $h$, which is called Beurling-Ahlfors extension. There is also another quasiconformal extension, called Douady-Earle extension, of $h$ to the unit disk which is conformally invariant (see $[\mathrm{DE}]$ ).

$\mathrm{Hu}$ and Shen [HS] introduced a integral operator $T_{h}^{-}$which is induced by the following kernel function

$$
\phi_{h}(\zeta, z)=\frac{1}{2 \pi i} \int_{S^{1}} \frac{h(w)}{(1-\zeta w)^{2}(1-z h(w))} d w, \quad(\zeta, z) \in \Delta \times \Delta,
$$

where $h$ is a sense preserving homeomorphism on the unit circle $S^{1}$. The function $\phi_{h}$ is holomorphic and also appeared in [Cui]. The integral operator $T_{h}^{-}$is defined as for any holomorphic function $\psi$ in $\Delta$,

$$
T_{h}^{-} \psi(\zeta)=\frac{1}{\pi} \iint_{\Delta} \phi_{h}(\zeta, z) \psi(\bar{z}) d x d y, \quad \zeta \in \Delta
$$

https://doi.org/10.5186/aasfm.2020.4502

2010 Mathematics Subject Classification: Primary 30C62; Secondary 30F60, 32G15.

Key words: Quasisymmetric homeomorphisms, Douady-Earle extension, integral operator, kernel functions, pull-back operator. 
Let $p \geq 2$. The Banach space $A^{p}$ consists of all holomorphic functions $\phi$ in the unit disk $\Delta$ with finite norm

$$
\|\phi\|_{A^{p}}=\left(\frac{1}{\pi} \iint_{\Delta}|\phi(z)|^{p}\left(1-|z|^{2}\right)^{p-2} d x d y\right)^{\frac{1}{p}}<\infty .
$$

When $p=2, A^{2}$ is a Hilbert space with inner product defined as

$$
\langle\phi, \psi\rangle=\frac{1}{\pi} \iint_{\Delta} \phi(z) \overline{\psi(z)} d x d y .
$$

We use $S H_{0}\left(S^{1}\right)$ to denote the set of all sense preserving homeomorphisms $h$ on the unit circle $S^{1}$, normalized by

$$
\frac{1}{2 \pi} \int_{S^{1}} h(\zeta)|d \zeta|=0
$$

For $w \in \Delta$, consider the Möbius transformation

$$
\Psi_{w}(\zeta)=\frac{\zeta-w}{1-\bar{w} \zeta}, \quad \zeta \in \Delta
$$

Let $h \in S H_{0}\left(S^{1}\right)$ and $H^{w}$ be the Poisson extension of $\Psi_{w} \circ h$. It is known that $H^{w}$ is a homeomorphism of $\bar{\Delta}$ onto $\bar{\Delta}$ for fix $w \in \Delta$ (see [Cho]). Let $p \geq 2$, we define kernel function $\mathfrak{H}_{h, p}$ as

$$
\mathfrak{H}_{h, p}(w)=\left(\frac{1}{2 \pi} \iint_{\Delta}\left|\bar{\partial} H^{w}(z)\right|^{p}\left(1-|z|^{2}\right)^{p-2} d x d y\right)^{1 / p}, \quad w \in \Delta .
$$

The function $\mathfrak{H}_{h, 2}$ already appeared in [Cui] and [HS].

We also consider the kernel function $\Phi_{h, p}$, which is defined as

$$
\Phi_{h, p}(w)=\left(\frac{1}{2 \pi} \iint_{\Delta}\left|\phi_{h}(z, w)\right|^{p}\left(1-|z|^{2}\right)^{p-2} d x d y\right)^{1 / p}, \quad w \in \Delta,
$$

where $p \geq 2$ and $h \in S H_{0}\left(S^{1}\right)$. The function $\Phi_{h, 2}$ has been used to study Teichmüller theory in [HS, SW, TS].

$\mathrm{Hu}$ and Shen [HS] proved the following result.

Theorem 1.2. [HS] Let $h \in S H_{0}\left(S^{1}\right)$. If $h$ is a quasisymmetric homeomorphism, then the integral operator $T_{h}^{-}: A^{2} \rightarrow A^{2}$ is bounded.

In this paper, we shall prove that the converse of Theorem 1.2 is also true and therefore obtain a characterization of quasisymmetric homeomorphism. Indeed, we prove the following general case.

Theorem 1.3. Let $h \in S H_{0}\left(S^{1}\right)$ and $p \geq 2$. Then the following statements are equivalent.

(i) $h$ is a quasisymmetric homeomorphism;

(ii) The integral operator $T_{h}^{-}: A^{p} \rightarrow A^{p}$ is bounded;

(iii) $\sup _{w \in \Delta} \mathfrak{H}_{h, p}(w)<\infty$;

(iv) $\sup _{w \in \Delta}\left(1-|w|^{2}\right) \Phi_{h, p}(w)<\infty$.

A sense preserving homeomorphism $h$ is called a symmetric homeomorphism if for any pair of adjacent sub-intervals $I_{1}$ and $I_{2}$ with $\left|I_{1}\right|=\left|I_{2}\right|$ in $S^{1}$, it holds that

$$
\frac{\left|h\left(I_{1}\right)\right|}{\left|h\left(I_{2}\right)\right|}=1+o(1), \quad\left|I_{1}\right|=\left|I_{2}\right| \rightarrow 0^{+} .
$$


The following result, due to Gardiner and Sullivan [GS], characterizes when a quasisymmetric homeomorphism $h$ is symmetric.

Theorem 1.4. [GS] A quasisymmetric homeomorphism $h$ is symmetric if and only if $h$ has a quasiconformal extension $f$ to the unit disk so that its complex dilataion $\mu=\partial_{\bar{z}} f / \partial_{z} f$ satisfies the property that $\mu(z) \rightarrow 0$ as $|z| \rightarrow 1$.

In terms of the integral operator $T_{h}^{-}$, kernel functions $\mathfrak{H}_{h, p}$ and $\Phi_{h, p}$, we obtain the following

Theorem 1.5. Let $p \geq 2$ and $h$ be a quasisymmetric homeomorphism on the unit circle $S^{1}$, normalized by (5). Then the following statements are equivalent.

(I) $h$ is a symmetric homeomorphism;

(II) The integral operator $T_{h}^{-}: A^{p} \rightarrow A^{p}$ is compact;

(III) $\lim _{|w| \rightarrow 1} \mathfrak{H}_{h, p}(w)=0$;

(IV) $\lim _{|w| \rightarrow 1}\left(1-\left|w^{2}\right|\right) \Phi_{h, p}(w)=0$.

Let $p \geq 2$, the Besov space $B_{p}\left(S^{1}\right)$ on the unit circle $S^{1}$ is the collection of measurable functions $f$ (modulo functions which are constant almost everywhere) for which the norm

$$
\|f\|_{p}=\left(\int_{0}^{2 \pi} \int_{0}^{2 \pi} \frac{\left|f\left(e^{i t}\right)-f\left(e^{i \theta}\right)\right|^{p}}{|t-\theta|^{2}} d t d \theta\right)^{1 / p}
$$

is finite (see $[\operatorname{Tr}]$ ). It is clear that the Besov space $B_{p}\left(S^{1}\right)$ is a Banach space and $B_{p}\left(S^{1}\right) \subset B_{q}\left(S^{1}\right)$ for $p \leq q$. $B_{2}\left(S^{1}\right)$ is the classic Sobolev space $H^{1 / 2}$ which consists of all integrable functions $u \in L^{1}([0,2 \pi])$ on the unit circle with semi-norm

$$
\|u\|_{p}=\left(\sum_{n=-\infty}^{+\infty}|n| \|\left. a_{n}(u)\right|^{2}\right)^{1 / 2},
$$

where $a_{n}(u)$ is the $n$-th Fourier coefficient of $u$, namely,

$$
a_{n}(u)=\frac{1}{2 \pi} \int_{0}^{2 \pi} u(\theta) e^{-i n \theta} d \theta
$$

Let $p \geq 2$. Recall that a sense preserving homeomorphism $h$ on the unit circle $S^{1}$ is $p$-integrable asymptotic affine homeomorphism if $h$ has a quasiconformal extension $f$ to the unit disk $\Delta$ whose complex dilatation $\mu$ satisfies

$$
\iint_{\Delta} \frac{|\mu(z)|^{p}}{\left(1-|z|^{2}\right)^{2}} d x d y<\infty
$$

The 2-integrable asymptotic affine homeomorphism was first introduced by Cui [Cui] and was much investigated in recent years (see [RSS1, RSS2, Shen, TT, STW]). For $p \geq 2$, the $p$-integrable asymptotic affine homeomorphism was first introduced and investigated by Guo [Guo] (see also [MY, Tang, Ya, HWS, TFS, TS]).

The authors proved the following result, which gives a intrinsic characteriztion of $p$-integrable asymptotic affine homeomorphism without using quasiconformal extension (see $[\mathrm{TS}])$.

Theorem 1.6. [TS] Let $p \geq 2$ and $h$ be a quasisymmetric homeomorphism, normalized by (5), on the unit circle $S^{1}$. Then $h$ is a $p$-integrable asymptotic affine homeomorphism if and only if $h$ is absolutely continuous (with respect to the arclength measure) such that $\log h^{\prime}$ belongs to $B_{p}\left(S^{1}\right)$. 
It should be pointed out that the case when $p=2$ of Theorem 1.6 was proved by Shen in [Shen].

We obtain a new characterization of $p$-integrable asymptotic affine homeomorphism by means of kernel functions $\mathfrak{H}_{h, p}$ and $\Phi_{h, p}$ in this paper.

Theorem 1.7. Let $p \geq 2$ and $h$ be a quasisymmetric homeomorphism, normalized by (5), on the unit circle $S^{1}$.Then the following statements are equivalent.

(a) $h$ is a p-integrable asymptotic affine homeomorphism;

(b) $\iint_{\Delta} \frac{\mathfrak{h}_{h, p}(w)^{p}}{\left(1-|w|^{2}\right)^{2}} d u d v<\infty$;

(c) $\iint_{\Delta} \Phi_{h, p}(w)^{p}\left(1-|w|^{2}\right)^{p-2} d u d v<\infty$.

We point out that the condition (c) in Theorem 1.7 is different from that in Theorem 3.4 in [TS], which is

$$
\iint_{\Delta} \Phi_{h, 2}(w)^{p}\left(1-|w|^{2}\right)^{p-2} d u d v<\infty
$$

The operator $T_{h}^{-}$is also related to the pull-back operator $T_{h}$ which is defined by

$$
T_{h}(f)=f \circ h, \quad f \in B_{p}\left(S^{1}\right),
$$

where $h \in S H_{0}\left(S^{1}\right)$. Vodop'yanov proved in [Vo] that the homeomorphism for which $T_{h}$ is a bounded operator on $B_{p}\left(S^{1}\right)(p \geq 2)$ is precisely quasisymmetric.

Theorem 1.8. [Vo] Let $p \geq 2$ and $h \in S H_{0}\left(S^{1}\right)$. Then $T_{h}$ is a bounded operator on $B_{p}\left(S^{1}\right)$ if and only if $h$ is a quasisymmetric homeomorphism.

Bourdaud and Sickel [BS] characterized the homeomorphisms for which $T_{h}$ is a bounded operator on $B_{p}\left(S^{1}\right)$ for the case when $1<p<\infty$. For the case when $p=2$, i.e., the Sobolev space $H^{1 / 2}$, Nag and Sullivan gave a different proof of Theorem 1.8 in [NS] and proved that the universal Teichmüller space can be embedded in the universal Siegel period matrix space by means of the pull-back operator $T_{h}$ (see also [TT]). This operator $T_{h}$ on $H^{1 / 2}$ has played an important role in the study of Teichmüller theory (see [HS, TT, SW, NS, Pa, Shen, STW, TS]).

By using Theorem 1.3, we shall give the "if" part of Theorem 1.8 a different proof.

We end this introduction section with the organization of the paper. In section 2 , we prove Theorem 1.3 by establishing a relationship between the complex dilatation of the Douady-Earle extension of $h \in S H_{0}\left(S^{1}\right)$ and the integral operator $T_{h}^{-}$. Section 3 is devoted to the proof of Theorems 1.5 and 1.7. We shall use Theorem 1.3 to study the pull-back operator $T_{h}$ in section 4 .

\section{Characterizations of quasisymmetric homeomorphisms}

In this section, we shall prove Theorem 1.3. Let us begin with some lemmas. The following result will prove very useful in our proof.

Lemma 2.1. [Zhu] Suppose that $(X, \mu)$ is a measure space and $K(x, y)$ is a nonnegative measurable function on $X \times X, K$ is the integral operator with kernel $K(x, y)$, that is

$$
K \varphi(z)=\iint_{X} K(x, y) \varphi(y) d \mu(y)
$$


Let $1<p<\infty$ with $1 / p+1 / q=1$. If there exist positive constant $C_{1}$ and $C_{2}$ and a positive measurable function $h$ on $X$ such that

$$
\iint_{X} K(x, y) h^{q}(y) d \mu(y) \leq C_{1} h^{q}(x)
$$

for almost every $x \in X$ and

$$
\iint_{X} K(x, y) h^{p}(x) d \mu(x) \leq C_{2} h^{p}(y)
$$

for almost every $y \in X$, then $K$ is a bounded operator on $L^{p}(X, d \mu)$ with norm less then or equal to $C_{1}^{1 / q} C_{2}^{1 / p}$.

See [Zhu] for a proof.

We also need the following integral estimates (see [Zhu]).

Lemma 2.2. [Zhu] Suppose that $z \in \Delta, s>0$ and $t>-1$. Then there exists constant $C>0$ so that

$$
\frac{1}{C} \frac{1}{\left(1-|z|^{2}\right)^{s}} \leq \iint_{\Delta} \frac{\left(1-|w|^{2}\right)^{t}}{|1-z \bar{w}|^{2+t+s}} d u d v \leq C \frac{1}{\left(1-|z|^{2}\right)^{s}}
$$

Let $h \in S H_{0}\left(S^{1}\right)$ and

$$
F(z, w)=\frac{1}{2 \pi} \int_{S^{1}} \frac{h(\zeta)-w}{1-\bar{w} h(\zeta)} \frac{1-|z|^{2}}{|\zeta-z|^{2}}|d \zeta|,
$$

where $(z, w) \in \Delta \times \Delta$. The Douady-Earle extension $E(h)$ of $h$ is defined as

$$
E(h)= \begin{cases}h(z), & \text { for } z \in S^{1}, \\ w, & \text { where } F(z, w)=0 \text { for } z \in \Delta,\end{cases}
$$

(see $[\mathrm{DE}])$.

The following result gives an estimate of the complex dilatation of the DouadyEarle extension $E(h)$ of $h \in S H_{0}\left(S^{1}\right)$ at the origin, which is needed in our proof of Theorem 1.3.

Lemma 2.3. Let $h \in S H_{0}\left(S^{1}\right)$ and $\nu$ be the complex dilatation of the DouadyEarle extension $E(h)$ of $h$. Then there exists a positive constant $C_{0}>0$ such that

$$
\frac{|\nu(0)|^{p}}{\left(1-|\nu(0)|^{2}\right)^{p / 2}} \leq C_{0} \frac{1}{2 \pi} \iint_{\Delta}|\bar{\partial} H(z)|^{p}\left(1-|z|^{2}\right)^{p-2} d x d y .
$$

Proof. The Fourier coefficient of $h$ are

$$
a_{n}=\frac{1}{2 \pi} \int_{-\pi}^{\pi} e^{-i n t} h\left(e^{i t}\right) d t, \quad n=0, \pm 1, \pm 2, \cdots
$$

It was shown (see [DE, Po, CZ]) that

$$
\begin{aligned}
& F_{z}(0,0)=\frac{1}{2 \pi} \int_{-\pi}^{\pi} e^{-i t} h\left(e^{i t}\right) d t=a_{1}, \\
& F_{\bar{z}}(0,0)=\frac{1}{2 \pi} \int_{-\pi}^{\pi} e^{i t} h\left(e^{i t}\right) d t=a_{-1}, \\
& F_{w}(0,0)=1, \\
& F_{\bar{w}}(0,0)=\frac{1}{2 \pi} \int_{-\pi}^{\pi} h\left(e^{i t}\right)^{2} d t=b .
\end{aligned}
$$


For simplicity of notations, we write $F_{z}$ for $F_{z}(0,0)$, etc. By a straight forward computation, we get

$$
\begin{aligned}
\bar{F}_{\bar{z}}+\bar{F}_{\bar{w}}{\overline{E(h)_{\bar{z}}}}+\bar{F}_{w} E(h)_{\bar{z}}=0, \\
F_{\bar{z}}+F_{\bar{w}}{\overline{E(h)_{\bar{z}}}}+F_{w} E(h)_{\bar{z}}=0 .
\end{aligned}
$$

Therefore, we have

$$
\frac{|\nu(0)|^{p}}{\left(1-|\nu(0)|^{2}\right)^{p / 2}}=\frac{\left|\overline{a_{1}} b+a_{-1}\right|^{p}}{\left(\left|a_{1}\right|^{2}-\left|a_{-1}\right|^{2}\right)^{p / 2}\left(1-|b|^{2}\right)^{p / 2}},
$$

where $\nu$ is the complex dilataion of $E(h)$. It is known that if $h$ is a sense-preserving homeomorphism on $S^{1}$, then there exists a positive constant $\delta>0$ so that

$$
\left|a_{1}\right|^{2}-\left|a_{-1}\right|^{2}=\delta>0, \quad 1-|b|^{2} \geq \frac{\delta^{2}}{4},
$$

(see $[\mathrm{Po}]$ ). Noting that $|h(\zeta)|=1$ for $|\zeta|=1$, we have $\left|a_{1}\right| \leq 1$. Consequently, combining (21) with (22) yields

$$
\frac{|\nu(0)|^{p}}{\left(1-|\nu(0)|^{2}\right)^{p / 2}} \leq \frac{\delta^{\frac{3 p}{2}}}{2^{p}}\left|\overline{a_{1}} b+a_{-1}\right|^{p} \leq \delta^{\frac{3 p}{2}}\left(\left|a_{-1}\right|^{p}+|b|^{p}\right) .
$$

We borrow some ideas from [Cui] to estimate $\left|a_{-1}\right|$ and $|b|$. Let $H$ be the Poisson extension of $h$. Observing that $H(0)=0$ and using Cauchy-Green formula to the function $z H(z)$, we obtain

$$
\left|a_{-1}\right|=\left|\frac{1}{2 \pi i} \int_{S^{1}} \frac{\zeta h(\zeta)}{\zeta-0} d \zeta\right|=\left|\frac{1}{2 \pi} \iint_{\Delta} \bar{\partial} H(z) d x d y\right|
$$

It follows from the Hölder inequality that there exists a constant $C_{1}>0$ so that

$$
\begin{aligned}
\left|a_{-1}\right|^{p} & \leq \frac{1}{2 \pi} \iint_{\Delta}|\bar{\partial} H(z)|^{p}\left(1-|z|^{2}\right)^{p-2} d x d y\left(\frac{1}{2 \pi} \iint_{\Delta}\left(1-|z|^{2}\right)^{\frac{2-p}{p-1}} d x d y\right)^{p-1} \\
& \leq C_{1} \frac{1}{2 \pi} \iint_{\Delta}|\bar{\partial} H(z)|^{p}\left(1-|z|^{2}\right)^{p-2} d x d y .
\end{aligned}
$$

Similarly, using Cauchy-Green formula to the function $H^{2}(z)$, we deduce that there exists a constant $C_{2}>0$ such that

$$
|b|=\left|\frac{1}{2 \pi i} \int_{S^{1}} \frac{h^{2}(\zeta)}{\zeta-0} d \zeta\right|=\left|\frac{1}{2 \pi} \iint_{\Delta} \frac{2 H(z)}{z} \bar{\partial} H(z) d x d y\right| \leq C_{2}\left|\frac{1}{2 \pi} \iint_{\Delta} \bar{\partial} H(z) d x d y\right| .
$$

By using the Hölder inequality agian and arguing similar to (24), we deduce that there exists a constant $C_{3}>0$ so that

$$
|b|^{p} \leq C_{3} \frac{1}{2 \pi} \iint_{\Delta}|\bar{\partial} H(z)|^{p}\left(1-|z|^{2}\right)^{p-2} d x d y .
$$

Therefore, it follows from (23), (24) and (25) that there exists a constant $C_{4}>0$ such that

$$
\frac{|\nu(0)|^{p}}{\left(1-|\nu(0)|^{2}\right)^{p / 2}} \leq C_{4} \frac{1}{2 \pi} \iint_{\Delta}|\bar{\partial} H(z)|^{p}\left(1-|z|^{2}\right)^{p-2} d x d y .
$$

The proof follows.

We point out that Lemma 2.3 is an extension of Proposition 7 in [CZ], where $p=2$ and $h$ is assumed to be a quasisymmetric homeomorphism on the unit circle $S^{1}$.

Now, we start our proof of Theorem 1.3. 
Proof of Theorem 1.3. It is known that

$$
\bar{\partial} H^{w_{0}}(z)=\left(1-\left|w_{0}\right|^{2}\right) \phi_{h}\left(\bar{z}, w_{0}\right)
$$

see [Cui] and [HS]. This shows that (iii) $\Leftrightarrow$ (iv). Therefore, it remains to show that (i) $\Rightarrow$ (ii) $\Rightarrow$ (iii) $\Rightarrow$ (i).

We first show that (i) $\Rightarrow$ (ii). Assume that $h$ is a quasisymmetric homeomorphism on the unit circle $S^{1}$, normalized by (5), and $f$ is the Beurling-Ahlfors extension of $h$ into $\Delta$. Let $\zeta \in \Delta$, by a result of [HS], we have

$$
T_{h}^{-} \psi(\zeta)=\frac{1}{\pi} \iint_{\Delta} \frac{\bar{\partial} f(w) \psi(f(w))}{(1-\zeta w)^{2}} d u d v
$$

Thus,

$$
\begin{aligned}
\left\|T_{h}^{-} \psi\right\|_{A^{p}}^{p}= & \frac{1}{\pi} \iint_{\Delta}\left|\frac{1}{\pi} \iint_{\Delta} \frac{\bar{\partial} f(w) \psi(f(w))}{(1-\zeta w)^{2}} d u d v\right|^{p}\left(1-|\zeta|^{2}\right)^{p-2} d \xi d \eta \\
\leq & \frac{1}{\pi^{p+1}} \iint_{\Delta}\left|\iint_{\Delta} \frac{\left(1-|w|^{2}\right)^{2-p}|\bar{\partial} f(w) \psi(f(w))|}{|1-\zeta w|^{2}}\left(1-|w|^{2}\right)^{p-2} d u d v\right|^{p} \\
& \cdot\left(1-|\zeta|^{2}\right)^{p-2} d \xi d \eta .
\end{aligned}
$$

Let $d \mu(w)=\left(1-|w|^{2}\right)^{p-2} d u d v$ and

$$
K(\zeta, w)=\frac{\left(1-|w|^{2}\right)^{2-p}}{|1-\zeta w|^{2}}, \quad(\zeta, w) \in \Delta \times \Delta .
$$

Consider the test function $h(w)=\left(1-|w|^{2}\right)^{\frac{3}{2 p}-1}$. It follows from Lemma 2.2 that there exists constant $C_{1}>0$ such that

$$
\begin{aligned}
\iint_{\Delta} \frac{\left(1-|w|^{2}\right)^{2-p}}{|1-\zeta w|^{2}} h(w)^{q} d \mu(w) & =\iint_{\Delta} \frac{\left(1-|w|^{2}\right)^{\frac{3 q}{2 p}-q}}{|1-\zeta w|^{2}} d u d v \\
& \leq C_{1}\left(1-|w|^{2}\right)^{\frac{3}{2 p}-1}=C_{1} h(w)^{q} .
\end{aligned}
$$

On the other hand, by Lemma 2.2 again, we deduce that there exists constant $C_{2}>0$ so that

$$
\begin{aligned}
\iint_{\Delta} \frac{\left(1-|w|^{2}\right)^{2-p}}{|1-\zeta w|^{2}} h(\zeta)^{p} d \mu(\zeta) & =\iint_{\Delta} \frac{\left(1-|w|^{2}\right)^{2-p}\left(1-|\zeta|^{2}\right)^{-1 / 2}}{|1-\zeta w|^{2}} d \xi d \eta \\
& \leq C_{2}\left(1-|\zeta|^{2}\right)^{\frac{3}{2}-p}=C_{2} h(\zeta)^{p}
\end{aligned}
$$

Combining (30) with (31) and using Lemma 2.1, we deduce that the following operator

$$
K \varphi(\zeta)=\iint_{\Delta} K(\zeta, w) \varphi(\zeta) d \mu(w)
$$

is bounded on $L^{p}(\Delta, d \mu)$. Consequently, it follows from (28) that there exists constant $C_{3}>0$ such that

$$
\left\|T_{h}^{-} \psi\right\|_{A^{p}}^{p} \leq \frac{C_{3}}{\pi^{p+1}} \iint_{\Delta}|\bar{\partial} f(\zeta) \psi(f(\zeta))|^{p}\left(1-|\zeta|^{2}\right)^{p-2} d \xi d \eta .
$$

It is well known that the Beurling-Ahlfors extension $f$ is bilipschitz continuous with respect to the hyperbolic metric (see [A, Le]), that is

$$
\frac{1}{C_{3}^{\prime}}\left(1-|f(\zeta)|^{2}\right) \leq\left(1-|\zeta|^{2}\right) J_{f}^{1 / 2}(\zeta) \leq C_{3}^{\prime}\left(1-|f(\zeta)|^{2}\right)
$$


where $C_{3}^{\prime}$ is a positive constant depending only on the complex dilatation of $f$ and $J_{f}$ is the Jacobian of $f$. Let $g=f^{-1}$ and $\mu$ be the complex dilatation of $g$. By (32) and a change of variable, we obtain

$$
\begin{aligned}
& \left\|T_{h}^{-} \psi\right\|_{A^{p}}^{p} \\
& \leq \frac{C_{3}}{\pi^{p+1}} \iint_{\Delta} \frac{|\bar{\partial} f(\zeta)|^{p}}{\left(|\partial f(\zeta)|^{2}-\mid \overline{\left.\left.\partial f(\zeta)\right|^{2}\right)^{p / 2}}\right.}|\psi(f(\zeta))|^{p}\left(1-|\zeta|^{2}\right)^{p-2} J_{f}^{p / 2}(\zeta) d \xi d \eta \\
& \leq C_{4} \frac{1}{\pi} \iint_{\Delta} \frac{|\mu(w)|^{p}}{\left(1-|\mu(w)|^{2}\right)^{p / 2}}|\psi(w)|^{p}\left(1-|w|^{2}\right)^{p-2} d u d v \\
& \leq C_{4} \frac{1}{\pi} \frac{k^{p}}{\left(1-k^{2}\right)^{p / 2}}\|\psi\|_{A^{p}}^{p},
\end{aligned}
$$

where $k=\|\mu\|_{\infty}<1$ and $C_{4}$ is a positive constant depending only on the complex dilatation of $f$. This shows that (i) $\Rightarrow$ (ii).

We next prove that (ii) $\Rightarrow$ (iii). Let $h$ be a sense-preserving homeomorphism on $S^{1}$. Suppose that the operator $T_{h}^{-}: B_{p} \rightarrow B_{p}$ is bounded. For $w_{0} \in \Delta$, consider the function

$$
\psi_{w_{0}}(\zeta)=\frac{1-\left|w_{0}\right|^{2}}{\left(1-w_{0} \zeta\right)^{2}}
$$

By Lemma 2.2, we conclude that there exists a constant $C_{5}>0$ independing of $w_{0}$ such that

$$
\left\|\psi_{w_{0}}\right\|_{A^{p}}^{p}=\frac{1}{\pi} \iint_{\Delta} \frac{\left(1-\left|w_{0}\right|^{2}\right)^{p}\left(1-|\zeta|^{2}\right)^{p-2}}{\left|1-w_{0} \zeta\right|^{2 p}} d \xi d \eta \leq C_{5}<\infty .
$$

It was proved in [HS] that

$$
T_{h}^{-} \psi_{w_{0}}(\zeta)=\left(1-\left|w_{0}\right|^{2}\right) \phi_{h}\left(\zeta, w_{0}\right) .
$$

Noting that $T_{h}^{-}: B_{p} \rightarrow B_{p}$ is bounded, combining (36), (37) with (27), we deduce that there exists a constant $C_{6}>0$ so that

$$
\mathfrak{H}_{h, p}\left(w_{0}\right)^{p}=\left\|T_{h}^{-} \psi_{w_{0}}\right\|_{A^{p}}^{p} \leq C_{6}\left\|\psi_{w_{0}}\right\|_{A^{p}}^{p} \leq C_{5} C_{6}<\infty .
$$

This finishes the proof of (ii) $\Rightarrow$ (iii).

Finally, we show that (iii) $\Rightarrow$ (i). Suppose that $h \in S H_{0}\left(S^{1}\right)$ and $\sup _{w_{0} \in \Delta} \mathfrak{H}_{h, p}\left(w_{0}\right)^{p}$ $<\infty$. By Theorem 1.1, to show that $h$ is quasisymmetric, it is sufficient to show that $h$ has a quasiconformal extension to the unit disk $\Delta$. Indeed, we shall show that the Douady-Earle extension $E(h)$ of $h$ is a quasiconformal mapping of $\Delta$ onto $\Delta$.

It is well known that if $h$ is a sense-preserving homeomorphism on $S^{1}$, then $E(h)$ is a homeomorphism of $\bar{\Delta}$ onto $\bar{\Delta}$ (see [DE, Po]). We next estimate the complex dilatation of $E(h)$.

Let $\left(w_{0}, z_{0}\right) \in \Delta \times \Delta$ with $w_{0}=E(h)\left(z_{0}\right)$. Consider the following two Möbius transformations

$$
\Psi_{w_{0}}(\zeta)=\frac{\zeta-w_{0}}{1-\overline{w_{0}} \zeta}, \quad \Gamma_{z_{0}}(\zeta)=\frac{\zeta+z_{0}}{1+\overline{z_{0}} \zeta}, \quad \zeta \in \Delta .
$$

Let $\mathfrak{h}=\Psi_{w_{0}} \circ h \circ \Gamma_{z_{0}}$ and $\mathcal{H}$ be the Poisson extension of $\mathfrak{h}$. Noting that $\mathcal{H} \circ \Gamma_{z_{0}}^{-1}$ is harmonic, we have $\mathcal{H} \circ \Gamma_{z_{0}}^{-1}=H^{w_{0}}$, where $H^{w_{0}}$ is the Poisson extension of $\Psi_{w_{0}} \circ h$. Since the Douady-Earle extension is conformal invariant (see [DE]), we have

$$
E(\mathfrak{h})=\Psi_{w_{0}} \circ E(h) \circ \Gamma_{z_{0}} .
$$


Let $\mu$ be the complex dilatation of the converse $E(h)^{-1}$ of $E(h)$. A computation gives

$$
\iint_{\Delta}|\bar{\partial} \mathcal{H}(z)|^{p}\left(1-|z|^{2}\right)^{p-2} d x d y=\iint_{\Delta}\left|\bar{\partial} H^{w_{0}}(z)\right|^{p}\left(1-|z|^{2}\right)^{p-2} d x d y
$$

and

$$
\left|\nu_{\mathfrak{h}}(0)\right|=\left|\mu\left(w_{0}\right)\right|
$$

where $\nu_{\mathfrak{h}}$ is the complex dilatation of $E(\mathfrak{h})$. Consequently, applying Lemma 2.3 to the quasisymmetric homeomorphism $\mathfrak{h}$ yields

$$
\frac{\left|\mu\left(w_{0}\right)\right|^{p}}{\left(1-\left|\mu\left(w_{0}\right)\right|^{2}\right)^{p / 2}} \leq C_{4}^{\prime} \frac{1}{2 \pi} \iint_{\Delta}\left|\bar{\partial} H^{w_{0}}(z)\right|^{p}\left(1-|z|^{2}\right)^{p-2} d x d y,
$$

where $C_{4}^{\prime}$ is a positive constant which independs on $w_{0} \in \Delta$.

Let $\nu$ be the complex dilatation of $E(h)$. Observing that $\left|\nu\left(z_{0}\right)\right|=\left|\mu\left(w_{0}\right)\right|$, by the condition (iii), we get

$$
\sup _{z_{0} \in \Delta} \frac{\left|\nu\left(z_{0}\right)\right|^{p}}{\left(1-\left|\nu\left(z_{0}\right)\right|^{2}\right)^{p / 2}} \leq C_{4}^{\prime}<\infty .
$$

On the other hand, it is known that for $z_{0} \in \Delta$,

$$
\left|\partial E(h)\left(z_{0}\right)\right|^{2}-\left|\bar{\partial} E(h)\left(z_{0}\right)\right|^{2}>0,
$$

(see $[\mathrm{Po}, \mathrm{DE}])$. Therefore, we conclude that $\|\nu\|_{\infty}<1$. This implies $E(h)$ is a quasiconformal mapping of $\Delta$ onto $\Delta$. The proof follows.

\section{Characterizations of symmetric and $p$-integrable asymptotic affine homeomorphisms}

In this section, we shall prove Theorem 1.5 and Theorem 1.7, which give some characterizations of symmetric and $p$-integrable asymptotic affine homeomorphisms.

We first prove Theorem 1.5.

Proof of Theorem 1.5. We first prove that (I) $\Rightarrow$ (II). Suppose that $h$ is a symmetric homeomorphism. Then $h$ has a quasiconformal extension $f$ to $\Delta$ with complex dilatation $\mu$, which is bilipschitz continuous with respect to the hyperbolic metric and satisfies the property that for any $\varepsilon>0$, there exists a constant $r_{0}>0$ so that $|\mu(z)|<\varepsilon$ for all $|z|>r_{0}$ (see [GS]). Assume that $\left\{\psi_{n}\right\}_{n=1}^{\infty}$ is a bounded sequence of $A_{p}$ and converges to zero on any compact subset of $\Delta$. Thus, there exists $N_{0}>0$ so that for all $n>N_{0}$,

$$
\iint_{\mid w \leq r_{0}}\left|\psi_{n}(w)\right|^{p}\left(1-|w|^{2}\right)^{p-2} d u d v<\varepsilon .
$$

It follows from (34) that for all $n>N_{0}$,

$$
\left\|T_{h}^{-} \psi_{n}\right\|_{A^{p}}^{p} \leq \frac{C_{4}^{\prime \prime} k^{p}}{\pi\left(1-k^{2}\right)^{p / 2}} \varepsilon+\frac{C_{4}^{\prime \prime} \varepsilon^{p}}{\pi\left(1-\varepsilon^{2}\right)^{p / 2}}\left\|\psi_{n}\right\|_{A_{p}}
$$

where $k=\|\mu\|_{\infty}$ and $C_{4}^{\prime \prime}>0$ depends only on $k$. This implies that $\left\{T_{h}^{-} \psi_{n}\right\}_{n=1}^{\infty}$ converges to zero in $A_{p}$. Therefore, $T_{h}^{-}: A_{p} \rightarrow A_{p}$ is a compact operator.

We next prove that (II) $\Rightarrow$ (III). Consider the function $\psi_{w_{0}}$ as in (35), which tends to zero on any compact subset of $\Delta$ as $\left|w_{0}\right| \rightarrow 1$. Also, from (36), we have 
$\left\|\psi_{w_{0}}\right\|_{A_{p}}^{p}<C_{5}$, where $C_{5}$ is independent of $w_{0}$. Since $T_{h}^{-}: A_{p} \rightarrow A_{p}$ is compact, we get from the first equality of (38) that

$$
\lim _{\left|w_{0}\right| \rightarrow 1} \mathfrak{H}_{h, p}\left(w_{0}\right)=\lim _{\left|w_{0}\right| \rightarrow 1}\left\|T_{h}^{-} \psi_{w_{0}}\right\|_{A^{p}}=0 .
$$

We proceed to show that (III) $\Rightarrow(\mathrm{I})$. Let $E(h)$ be the Douady-Earle extension of $h$ with complex dilatation $\nu$. From (40) and the condition (III), we have

$$
\lim _{|w| \rightarrow 1}|\mu(w)|=0
$$

where $\mu$ is the complex dilatation of the converse $E(h)^{-1}$ of $E(h)$. Noting that $|\nu(z)|=|\mu(w)|$, where $E(h)(z)=w$, we get

$$
\lim _{|z| \rightarrow 1}|\nu(z)|=0 \text {. }
$$

Thus, we conclude from Theorem 1.4 that $h$ is symmetric.

Finally, it follows from (27) that (III) $\Leftrightarrow$ (IV). The proof of Theorem 1.5 is completed.

We next prove Theorem 1.7.

Proof of Theorem 1.7. It follows from (27) that (b) $\Leftrightarrow$ (c). We need only to show that (a) $\Leftrightarrow(\mathrm{b})$. Let $h$ be a $p$-integrable asymptotic affine homeomorphism on the unit circle $S^{1}$. Then $h$ has a quasiconformal extension $f$ to the unit disk $\Delta$ with complex dilatation $\mu$, which is bilipschitz continuous with respect to the hyperbolic metric and satisfies

$$
\iint_{\Delta} \frac{|\mu(z)|^{p}}{\left(1-|z|^{2}\right)^{2}} d x d y<\infty
$$

(see [Cui, Tang]). By applying Lemma 2.2, we conclude from (37), (27) and (34) that there exist two positive constants $C_{1}$ and $C_{2}$ so that

$$
\begin{aligned}
\iint_{\Delta} \frac{\mathfrak{H}_{h, p}(w)^{p}}{\left(1-|w|^{2}\right)^{2}} d u d v & \leq C_{1} \frac{1}{\pi} \iint_{\Delta} \iint_{\Delta} \frac{|\mu(z)|^{p}\left|\psi_{w}(z)\right|^{p}}{\left(1-|\mu(z)|^{2}\right)^{p / 2}} \frac{\left(1-|z|^{2}\right)^{p-2}}{\left(1-|w|^{2}\right)^{2}} d u d v d x d y \\
& \leq C_{2} \iint_{\Delta} \frac{|\mu(z)|^{p}}{\left(1-|z|^{2}\right)^{2}} d x d y<\infty
\end{aligned}
$$

where $\psi_{w}$ is defined as in (35). This finishes the proof of $(\mathrm{a}) \Rightarrow(\mathrm{b})$.

Conversely, we consider the Douady-Earle extension $E(h)$ of $h$ with complex dilatation $\nu$. From (40), we obtain

$$
\iint_{\Delta} \frac{|\mu(w)|^{p}}{\left(1-|w|^{2}\right)^{2}} d u d v<\infty
$$

where $\mu$ is the complex dilatation of the converse $E(h)^{-1}$ of $E(h)$. Noting that the set of all $p$-integrable asymptotic affine homeomorphisms on the unit circle $S^{1}$ is a group (see [Tang]), we conclude that

$$
\iint_{\Delta} \frac{|\nu(w)|^{p}}{\left(1-|w|^{2}\right)^{2}} d u d v<\infty .
$$

This completes the proof of Theorem 1.7.

Combing Theorem 1.7 and Theorem 1.6 gives the following 
Corollary 3.1. Let $p \geq 2$ and $h$ be a quasisymmetric homeomorphism, normalized by (5), on the unit circle $S^{1}$. Then $h$ is absolutely continuous (with respect to the arc-length measure) such that $\log h^{\prime}$ belongs to $B_{p}\left(S^{1}\right)$ if and only if

$$
\iint_{\Delta} \frac{\mathfrak{H}_{h, p}(w)^{p}}{\left(1-|w|^{2}\right)^{2}} d u d v<\infty
$$

\section{Pull-back operators indecued by quasisymmetric homeomorphisms}

In this section, we shall use Theorem 1.3 to prove the "if" part of Theorem 1.8. We first recall some notions. Let $p \geq 2$ and $D_{p}(\Delta)$ denote the space of all harmonic functions $u$ in the unit disk $\Delta$ with semi-norm

$$
\|u\|_{D_{p}}=\left(\frac{1}{\pi} \iint_{\Delta}(|\partial u(z)|+|\bar{\partial} u(z)|)^{p}\left(1-|z|^{2}\right)^{p-2} d x d y\right)^{\frac{1}{p}} .
$$

Let $H$ be the Poisson integral operator. It is well known that a integrable function $v$ on the unit circle $S^{1}$ belongs to the the Besov space $B_{p}\left(S^{1}\right)$ if and only if $H(v) \in$ $D_{p}(\Delta)$ and there is constant $C>0$ such that for any $v \in B_{p}\left(S^{1}\right)$,

$$
\frac{1}{C}\|v\|_{p} \leq\|H(v)\|_{D_{p}} \leq C\|v\|_{p}
$$

(see [Tr], [RS]). We denote by $D_{a}^{p}(\Delta)$ be the Banach space of all analytic functions $\varphi$ in $\Delta$ with the semi-norm

$$
\|u\|_{D_{a}^{p}}=\left(\frac{1}{\pi} \iint_{\Delta}\left|\varphi^{\prime}(z)\right|^{p}\left(1-|z|^{2}\right)^{p-2} d x d y\right)^{\frac{1}{p}} .
$$

Then it is clear that $D_{p}(\Delta)=D_{a}^{p}(\Delta) \oplus \overline{D_{a}^{p}(\Delta)}$, precisely, for each $u \in D_{a}^{p}(\Delta)$, there exists a unique pair of holomorphic functions $\varphi$ and $\psi$ in $D_{a}^{p}(\Delta)$ with $\varphi(0)-u(0)=$ $\psi(0)=0$ such that $u=\varphi+\bar{\psi}$. Define two operator $P^{+}$and $P^{-}$by $P^{+} u=\varphi$ and $P^{-} u=\overline{\psi(\bar{z})}$. Let $h \in S H_{0}\left(S^{1}\right)$, we define two further operators $P_{h}^{+}=P^{+} \circ H \circ T_{h}$ and $P_{h}^{-}=P^{-} \circ H \circ T_{h}$.

We state the "if" part of Theorem 1.8 as following

Theorem 4.1. Let $2 \leq p<\infty$ and $h \in S H_{0}\left(S^{1}\right)$. If $h$ is a quasisymmetric homeomorphism, then $T_{h}: B_{p}\left(S^{1}\right) \rightarrow B_{p}\left(S^{1}\right)$ is a bounded operator.

Proof. To prove Theorem 4.1, we need another integral operator, which is defined by the following kernel function

$$
\psi_{h}(\zeta, z)=\frac{1}{2 \pi i} \int_{S^{1}} \frac{h(w)}{(\zeta-w)^{2}(1-z h(w))} d w, \quad(\zeta, z) \in \Delta \times \Delta .
$$

The integral operator $T_{h}^{+}$is defined as

$$
T_{h}^{+} \psi(\zeta)=\frac{1}{\pi} \iint_{\Delta} \psi_{h}(\zeta, z) \psi(\bar{z}) d x d y, \quad \psi \in A_{p}, \quad \zeta \in \Delta .
$$

Let $\zeta \in \Delta$ and $f$ be the Beurling-Ahlfors extension of $h$ into $\Delta$, by a result of [HS], we have

$$
T_{h}^{+} \psi(\zeta)=\frac{1}{\pi} \iint_{\Delta} \frac{\partial f(w) \psi(f(w))}{(1-\zeta \bar{w})^{2}} d u d v
$$


Thus,

$$
\begin{aligned}
\left\|T_{h}^{+} \psi\right\|_{A^{p}}^{p}= & \frac{1}{\pi} \iint_{\Delta}\left|\frac{1}{\pi} \iint_{\Delta} \frac{\partial f(w) \psi(f(w))}{(1-\zeta \bar{w})^{2}} d u d v\right|^{p}\left(1-|\zeta|^{2}\right)^{p-2} d \xi d \eta \\
\leq & \frac{1}{\pi^{p+1}} \iint_{\Delta}\left|\iint_{\Delta} \frac{\left(1-|w|^{2}\right)^{2-p}|\partial f(w) \psi(f(w))|}{|1-\zeta \bar{w}|^{2}}\left(1-|w|^{2}\right)^{p-2} d u d v\right|^{p} \\
& \cdot\left(1-|\zeta|^{2}\right)^{p-2} d \xi d \eta .
\end{aligned}
$$

Let $d \mu(w)=\left(1-|w|^{2}\right)^{p-2} d u d v$ and

$$
K_{1}(\zeta, w)=\frac{\left(1-|w|^{2}\right)^{2-p}}{|1-\zeta \bar{w}|^{2}}, \quad(\zeta, w) \in \Delta \times \Delta .
$$

Consider the test function $h(w)=\left(1-|w|^{2}\right)^{\frac{3}{2 p}-1}$. By the same method as in Theorem 1.3, we can deduce that the following operator

$$
K_{1} \varphi(\zeta)=\iint_{\Delta} K_{1}(\zeta, w) \varphi(\zeta) d \mu(w)
$$

is bounded on $L^{p}(\Delta, d \mu)$. Thus, we conclude from (48) that there exists constant $C_{1}^{\prime}>0$ so that

$$
\left\|T_{h}^{+} \psi\right\|_{A^{p}}^{p} \leq \frac{C_{1}^{\prime}}{\pi^{p+1}} \iint_{\Delta}|\partial f(\zeta) \psi(f(\zeta))|^{p}\left(1-|\zeta|^{2}\right)^{p-2} d \xi d \eta .
$$

Let $J_{f}$ be the Jacobian of $f$ and $\mu$ the complex dilatation of $g=f^{-1}$. By (50), (33) and a change of variable, we conclude that there exists constant $C_{2}^{\prime}>0$ so that

$$
\begin{aligned}
& \left\|T_{h}^{+} \psi\right\|_{A^{p}}^{p} \\
& \leq \frac{C_{1}^{\prime}}{\pi^{p+1}} \iint_{\Delta} \frac{|\partial f(\zeta)|^{p}}{\left(|\partial f(\zeta)|^{2}-|\bar{\partial} f(\zeta)|^{2}\right)^{p / 2}}|\psi(f(\zeta))|^{p}\left(1-|\zeta|^{2}\right)^{p-2} J_{f}^{p / 2}(\zeta) d \xi d \eta \\
& \leq C_{2}^{\prime} \frac{1}{\pi} \iint_{\Delta} \frac{1}{\left(1-|\mu(w)|^{2}\right)^{p / 2}}|\psi(w)|^{p}\left(1-|w|^{2}\right)^{p-2} d u d v \\
& \leq C_{2}^{\prime} \frac{1}{\pi} \frac{1}{\left(1-k^{2}\right)^{p / 1}}\|\psi\|_{A^{p}}^{p}
\end{aligned}
$$

where $k=\|\mu\|_{\infty}<1$.

It is cleat that $D \varphi(z)=\varphi^{\prime}(z)$ determines an isometric isomorphism from $D_{a}^{p}(\Delta)$ onto $A^{p}$. By the same reasoning as in the proof of Theorem 3.1 in [HS], we can show that on $D_{a}^{p}(\Delta)$,

$$
D \circ P_{h}^{+}=T_{h}^{+} \circ D, \quad D \circ P_{h}^{-}=T_{h}^{-} \circ D .
$$

Thus, we conclude from (34), (51) and (52) that $P_{h}^{+}: D_{a}^{p}(\Delta) \rightarrow D_{a}^{p}(\Delta)$ and $P_{h}^{-}: D_{a}^{p}(\Delta)$ $\rightarrow D_{a}^{p}(\Delta)$ are bounded operators.

Let $u \in B_{p}\left(S^{1}\right)$ and $H(u)=\varphi+\bar{\psi}$. Observe that

$$
\begin{aligned}
H \circ T_{h} u(z) & =H \circ T_{h} \varphi(z)+\overline{H \circ T_{h} \psi(z)} \\
& =P_{h}^{+} \varphi(z)+P_{h}^{-} \varphi(\bar{z})+\overline{P_{h}^{+} \psi(z)}+\overline{P_{h}^{-} \psi(\bar{z})} .
\end{aligned}
$$

We conclude from the discussions above that there exist positive constant $C_{3}^{\prime}, C_{4}^{\prime}, C_{5}^{\prime}$ such that

$$
\left\|T_{h} u\right\|_{p} \leq C_{3}^{\prime}\left\|H \circ T_{h} u\right\|_{D_{p}} \leq C_{4}^{\prime}\left(\|\varphi\|_{D_{a}^{p}}+\|\psi\|_{D_{a}^{p}}\right) \leq C_{5}^{\prime}\|u\|_{p} .
$$

The proof follows. 
Acknowledgements. This work was supported by National Natural Science Foundation of China (Grant Nos. 11601100) and the foundation of Guizhou Provincial Science and Technology Department (Grant Nos. [2017]7337, [2017]5726).

\section{References}

[A] Ahlfors, L. V.: Lecture on quasiconformal mappings. - Princeton, Van Nostrand, New Jersey, 1966.

[BA] Beurling, A., and L. V. Ahlfors: The boundary correspondence under quasiconformal mappings. - Acta Math. 96, 1956, 125-142.

[BS] Bourdaud, G., and W. Sickel: Changes of variable in Besov spaces. - Math. Nachr. 198, 1999, 19-39.

[Cho] Choquet, G.: Sur un type de transformation analytique généralisant la représentation conforme et défine au moyen de fonctions harmoniques. - Bull. Sci. Math. 69, 1945, 156165.

[Cui] CuI, G.: Integrably asymptotic affine homeomorphisms of the circle and Teichmüller spaces. - Sci. China Ser. A 43, 2000, 267-279.

[CZ] Cui, G., and M. Zinsmeister: BMO-Teichmüller spaces. - Illinois J. Math. 48, 2004, 1223-1233.

[DE] Doundy, A., and C. EARle: Conformally natural extension of homeomorphisms of the circle. - Acta Math. 157, 1986, 23-48.

[Guo] Guo, H.: Integrable Teichmüller spaces. - Sci. China Ser. A 43, 2000, 47-58.

[GS] Gardiner, F. P., and D. Sullivan: Symmetric structures on a closed curve. - Amer. J. Math. 114, 1992, 683-736.

[HWS] He, Y., H. Wei, and Y. Shen: Some notes on quasisymmetric flows of Zygmund vector fields. - J. Math. Anal. Appl. 455, 2017, 370-380.

[HS] Hu, Y., and Y. Shen: On quasisymmetric homeomorphisms. - Israel J. Math. 191, 2012, 209-226.

[Le] Lehto, O.: Univalent functions and Teichmüller spaces. - Springer-Verlag, New York, 1986.

[MY] Matsuzaki, K., and M. Yanagishita: Asymptotic conformality of the barycentric extension of quasiconformal maps. - Filomat 31, 2017, 85-90.

[Na] NAG, S.: The complex analytic theory of Teichmüller space. - Wiley-Interscience, 1988.

[NS] NAG, S., and D. Sullivan: Teichmüller theory and the universal period mapping via quantum calculus and the $H^{1 / 2}$ space on the circle. - Osaka J. Math. 32, 1995, 1-34.

[Pa] PARTYKA, D.: The generalized Neumann-Poincaré operator and its spectrum. - Dissertations Math. 484, Institute of Mathematics, Polish Academy of Sciences, Warszawa, 1997.

[Po] Pommerenke, Ch.: Boundary behaviour of conformal maps. - Springer-Verlag, Berlin, 1992.

[RS] RUNSt, T., and W. SickeL: Sobolev spaces of fractional order, Nemytskij operators, and nonlinear partial differential equations. - De Gruyster Series in Nonlinear Analysis and Applications 3, Walter de Gruyter, 1996.

[RSS1] Radnell, D., E. Schippers, and W. Staubach: A Hilbert manifold structure on the Weil-Petersson class Teichmüller space of bordered Riemann surfaces. - Commun. Contemp. Math. 17:42, 2015, 1550016, 1-42.

[RSS2] Radnell, D., E. Schippers, and W. Staubach: Convergence of the Weil-Petersson metric on the Teichmüller spaces of bordered Riemann surfaces. - Commun. Contemp. Math. 19:1, 2017, 1650025, 1-39.

[Shen] Shen, Y.: Weil-Peterssen Teichmüller space. - Amer. J. Math. 140, 2018, 1041-1074. 
[STW] Shen, Y., S. Tang, and L. Wu: Weil-Petersson and little Teichmüller spaces on the real line. - Ann. Acad. Sci. Fenn. Math. 43, 2018, 935-43.

[SW] Shen, Y., and H. WeI: Universal Teichmüller space and BMO. - Adv. Math. 234, 2013, $129-148$.

[Tang] TANG, S.: Some characterizations of the integrable Teichmüller space. - Sci. China Math. $56,2013,541-551$.

[TFS] Tang, S., X. Feng, and Y. Shen: Besov functions and the tangent space to the integral Teichmüller space. - Chinese Ann. Math. Ser. B 39, 2018, 963-972.

[Tr] Triebel, H.: Theory of function spaces. - Monographs in Mathematics 78, Birkhäuser, Basel, 1983.

[TT] Takhtajan, L., and L. P. TeO: Weil-Petersson metric on the universal Teichmüller space. - Mem. Amer. Math. Soc. 183, 2006, 1-119.

[TS] Tang, S., and Y. Shen: Integrable Teichmüller space. - J. Math. Anal. Appl. 465, 2018, 658-672.

[Vo] Vodop'Yanov, S. K.: Mappings of homogeneous groups and embeddings of function spaces. - Sibirsk. Mat. Zh. 30, 1989, 25-41.

[Ya] Yanagishita, M.: Introduction of a complex structure on the $p$-integrable Teichmüller space. - Ann. Acad. Sci. Fenn. Math. 39, 2014, 947-971.

[Zhu] ZHu, K.: Operator theory in function spaces. Second edition. - Math. Surveys Monogr. 138, Amer. Math. Soc., Providence, RI, 2007.

Received 23 October 2018 • Accepted 1 February 2019 